\title{
Integrated database for identifying candidate genes for Aspergillus flavus resistance in maize
}

\author{
Rowena Y Kelley ${ }^{1,3^{*}}$, Cathy Gresham², Jonathan Harper ${ }^{2,3}$, Susan M Bridges ${ }^{2,3}$, Marilyn L Warburton ${ }^{4}$, \\ Leigh K Hawkins ${ }^{4}$, Olga Pechanova ${ }^{1}$, Bela Peethambaran ${ }^{5}$, Tibor Pechan ${ }^{6}$, Dawn S Luthe ${ }^{7}$, J E Mylroie ${ }^{1}$, \\ Arunkanth Ankala ${ }^{8}$, Seval Ozkan" ${ }^{9}$ W B Henry ${ }^{4}$, W P Williams ${ }^{4}$
}

From Seventh Annual MCBIOS Conference. Bioinformatics: Systems, Biology, Informatics and Computation Jonesboro, AR, USA. 19-20 February 2010

\begin{abstract}
Background: Aspergillus flavus Link:Fr, an opportunistic fungus that produces aflatoxin, is pathogenic to maize and other oilseed crops. Aflatoxin is a potent carcinogen, and its presence markedly reduces the value of grain. Understanding and enhancing host resistance to A. flavus infection and/or subsequent aflatoxin accumulation is generally considered an efficient means of reducing grain losses to aflatoxin. Different proteomic, genomic and genetic studies of maize (Zea mays L.) have generated large data sets with the goal of identifying genes responsible for conferring resistance to $A$. flavus, or aflatoxin.

Results: In order to maximize the usage of different data sets in new studies, including association mapping, we have constructed a relational database with web interface integrating the results of gene expression, proteomic (both gel-based and shotgun), Quantitative Trait Loci (QTL) genetic mapping studies, and sequence data from the literature to facilitate selection of candidate genes for continued investigation. The Corn Fungal Resistance Associated Sequences Database (CFRAS-DB) (http://agbase.msstate.edu/) was created with the main goal of identifying genes important to aflatoxin resistance. CFRAS-DB is implemented using MySQL as the relational database management system running on a Linux server, using an Apache web server, and Perl CGI scripts as the web interface. The database and the associated web-based interface allow researchers to examine many lines of evidence (e.g. microarray, proteomics, QTL studies, SNP data) to assess the potential role of a gene or group of genes in the response of different maize lines to A. flavus infection and subsequent production of aflatoxin by the fungus.

Conclusions: CFRAS-DB provides the first opportunity to integrate data pertaining to the problem of $A$. flavus and aflatoxin resistance in maize in one resource and to support queries across different datasets. The web-based interface gives researchers different query options for mining the database across different types of experiments. The database is publically available at http://agbase.msstate.edu.
\end{abstract}

\section{Background}

Mycotoxins are considered to be among the most significant food contaminants because of their negative impact on public health, food security, and the national economy of many countries. They affect a wide range of agricultural products, including cereals, nuts, and

\footnotetext{
* Correspondence: rkelley@bch.msstate.edu

'Department of Biochemistry and Molecular Biology, Mississippi State University, MS, USA

Full list of author information is available at the end of the article
}

oilseeds, that are used for human food and animal feeds. Mycotoxin contamination of susceptible commodities occurs as a result of environmental conditions in the field as well as improper harvesting, storage, and processing operations [1-3]. Mycotoxins may be carcinogenic, mutagenic, teratogenic, and immunosuppressive. Estimated annual losses in the USA and Canada arising from the impact of mycotoxins on the feed and livestock industries are of the order of $\$ 5$ billion. In developing countries, food staples are susceptible to contamination

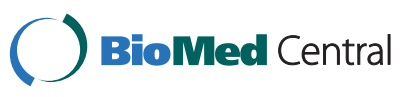

() 2010 Kelley et al; licensee BioMed Central Ltd. This is an open access article distributed under the terms of the Creative Commons Attribution License (http://creativecommons.org/licenses/by/2.0), which permits unrestricted use, distribution, and reproduction in any medium, provided the original work is properly cited. 
and it is likely that a significant number of human deaths are attributable to the consumption of mycotoxins [1].

Aflatoxins are an important group of mycotoxins that are produced as secondary metabolites under conducive climatic conditions by the fungi Aspergillus flavus Link: Fr. and A. parasiticus Speare [3]. The most common aflatoxins produced by these fungi are B1, B2, G1, and G2; the most potent of these is B1 [4,5]. Because aflatoxin induces animal diseases, particularly liver cancer in humans, aflatoxins are the most widely studied mycotoxins. More than 50 countries have established or proposed regulations for controlling aflatoxin ingestion from contaminated foods and feeds [6-9]. The U.S. Food and Drug Administration [10] set a tolerance level of 20 $\mathrm{ng} \mathrm{g}^{-1}$ for aflatoxin B1 in maize (Zea mays L.). Grain that exceeds that level cannot be shipped via interstate commerce or used for human consumption. As of 2003, 61 countries have imposed regulations on aflatoxin B1 levels in foodstuffs [11]. Aflatoxin contamination of corn reached epidemic proportions in the U.S. in 1977 and 1998. In 1998, aflatoxin contamination resulted in $\$ 85$ to $\$ 100$ million in losses to maize producers in Texas, Louisiana, and Mississippi [12], and the combined crop loss due to aflatoxin epidemics in the southern USA during 1988, 1989, 1995, 1996, and 1998 surpassed $\$ 1$ billion [13]. Because A. flavus infection and aflatoxin accumulation can lead to substantial economic losses and risk to human and animal health, efforts to create resistant corn varieties are ongoing. The seriousness of the problem of aflatoxin has generated large quantities of data from many breeding and genetics, fungal biology, physiological and biochemical studies, but the problem has yet to be solved.

Genetics and breeding studies have focused on identifying lines with genetic resistance, either to fungal infection or specifically to aflatoxin accumulation [14-16], and mapping the resistance genes and moving them into susceptible corn inbred lines. Biochemical studies have worked to identify the aflatoxin toxicity pathways and determine its effects on the physiology of the animals that eat infected grain and ways to mitigate these effects $[4,5]$. Genomics and proteomics studies of both the fungus and the corn plant, seek to identify pathways, proteins, and genes involved in toxin metabolism and catabolism, to manipulate the production and reduction of aflatoxin and keep levels low in fungal-infected grains. Finally, many studies of resistance mechanisms to fungal infection and spread in plants are available, and much of this research may shed some light on the search for genes conferring resistance to A. flavus.

The Corn Fungal Resistance Associated Sequences Database (CFRAS-DB) was created to integrate different data types from studies of aflatoxin resistance in maize by different researchers to allow us to more effectively investigate mechanisms, pathways, and genes that confer resistance to some maize lines, and allow the development of high yielding maize lines with resistance to $A$. flavus and/or aflatoxin. CFRAS-DB uses a MySQL relational database management system with dynamic query and data integration to help researchers identify gene sequences with a possible role in aflatoxin accumulation resistance, using multiple lines of evidence. This may help narrow the number of potential candidate genes that will be studied to determine or confirm function in aflatoxin accumulation resistance in maize. Genes with the highest probability of being associated with aflatoxin accumulation resistance should be given priority for further study, including the creation of transgenic plants to test construct effect, association analysis of candidate genes with important phenotypes, or other biochemical or genetic tests of gene function.

One of the major challenges of integrating data from different types of experiments conducted by different researchers over several different years is mapping the identifiers used in the different studies to a common set of identifiers. MaizeSequence.org is a central resource for the recently sequenced maize genome and we use their gene model identifiers as reference identifiers. Sequences from all experiments stored in CFRAS-DB are mapped to MaizeSequence.org sequences and the MaizeSequence identifiers serve as the primary key linking the different experiments. Data entries may represent DNA sequences, protein sequences, genetic loci (locations) on a chromosome, expression data of specific proteins or RNA sequences, or physiological or phenotypic traits linked to genetic markers at known loci. This database supports multiple dynamic queries to find and display data for different genes and genotypes (individual maize plants). The outputs based on these queries can also be downloaded and used as input for pathway analysis programs for more evidence of a common function for genes these queries identify.

\section{Results and discussion}

CFRAS-DB integrates information from the newly sequenced maize genome [17] with results and annotation efforts from the aflatoxin host plant resistance community to help researchers identify genes that confer resistance in maize to $A$. flavus. Table 1 provides a list of the community datasets stored in CFRAS-DB and Table 2 provides a list of external resources integrated into CFRAS-DB. The resistance data has been collected using a wide variety of techniques, different identifiers have been used to specify genes/proteins in the experimental results from different studies, and because the newly released draft maize genome is still quite immature and will be updated on a regular basis. In addition, 
Table 1 Types of data currently available from the maize/Aspergillus research community and the type of identifier reported

\begin{tabular}{llll}
\hline Data Type & Identifier Type & $\begin{array}{l}\text { Number of } \\
\text { Records }\end{array}$ & Data Source \\
\hline $\begin{array}{l}\text { Gene } \\
\text { microarrays }\end{array}$ & $\begin{array}{l}\text { ESTs with a variety of } \\
\text { identifiers }\end{array}$ & 62,178 & MSU 2002 Kelley 2DAI MSU 2005 Kelley 4 DAI \\
$\begin{array}{l}\text { Shotgun } \\
\text { proteomics }\end{array}$ & UniProtKB & 512 & MSU 2004 Pechanova Rachis \\
$\begin{array}{l}\text { Gel based } \\
\text { proteomics } \\
\text { QTL studies }\end{array}$ & UniProtKB & 411 & MSU 2004 Pechanova Rachis \\
BNP studies & EST identifiers & 67 & $\begin{array}{l}\text { Paul et al 2003 Widstrom et al 2003 Busboom 2004 Unpublished QTLBrooks 2005 } \\
\text { Warburton 2009 Warburton 2010 }\end{array}$ \\
\hline
\end{tabular}

the resistance studies have used a variety of different maize genotypes (most are different from the B73 genotype used for the maize sequencing project) and a variety of A.flavus genotypes. These issues motivated a gene centric design where all entities are mapped to MaizeSequence identifiers. This approach enables data mining across multiple experiments and easy integration with AgBase. Figure 1 shows the entity-relationship diagram for the core CFRAS-DB tables.

The database now supports queries that enable users to identify genes implicated in resistance by multiple lines of evidence and that are the most promising for future investigations. The web-interface provides the user with the capability to generate many additional queries. For example, Figure 2 shows the search interface for gene expression results. The user can select one or more experiments, genotypes, bins, direction of gene regulation, and/or specific identifiers. Figure 3 shows the results for a gene expression query where the experiment was MSU 2005 Kelley 4 DAI, the genotype is VA35, bins are 1.00-1.02, and gene regulation is "all differentially expressed genes." The results page allows the user to sort the data on any column where the header is underlined (all columns in this example). Our goal is to allow the user to access the data easily, to any desired level of detail, and from many different points of view. If the user clicks on a sequence identifier in a gene expression list, information about the gene from MaizeSequence is displayed (Figure 4). If the user selects an EST id, a list of all the experimental and mapping data for the corresponding MaizeSequence gene model will be displayed. From the expression results (Figure 3) one can also select a bin link. The resulting bin data display includes QTLs that fall within the bin and a list of all sequences in the bin for which experimental data is available. From the gene expression results page, one can also explore detailed metadata about each experiment and a description of the genotypes used in the experiments. Table 3 provides a list of all queries currently available. Query capabilities will be extended and refined to meet user needs as the database expands. CFRAS-DB results tables can be downloaded as Excel worksheets for further analysis.

Examples of candidate genes that have been identified from multiple lines of evidence in CFRAS-DB include AW261420: Formate dehydrogenase Zea mays (Va35), AW438153: DRE binding factor 1 (dbf1) (Mp313E), TC239060: Pathogenesis-related protein 1 precursor (Va35), and AZM4_24463: Disease resistance protein RPM1 (Mp313E). Sequencing has been performed on some candidates to find single nucleotide polymorphisms (SNP) and insertion / deletions (indels) in the target genes for further validation and possible use in developing markers for resistance to aflatoxin accumulation in maize. These SNPs are available in CFRAS-DB.

\section{Materials and methods}

CFRAS-DB is implemented in MySQL 5.1.31-community version and resides on a Quad Core XEON processor X5355 2.66 GHz 2664 MHz running SUSE Linux.

Table 2 External data sources integrated into CFRAS-DB

\begin{tabular}{lll}
\hline External Source & Number of Records & Data Type \\
\hline ZMGI & 21,900 & EST sequences with ids from a variety of sources \\
TIGR & 5,071 & Assembled EST sequences (TCS) with TC ids \\
NCBI & 1,101 & EST sequences and detailed gene information \\
MaizeGDB & 25,943 & Bin location for EST identifiers and genes \\
UniProtKB & 19,006 & UniProtKB protein identifiers and identifier mapping \\
MaizeSequence & 16,955 & Genome sequence, gene models, gene transcript mapping
\end{tabular}




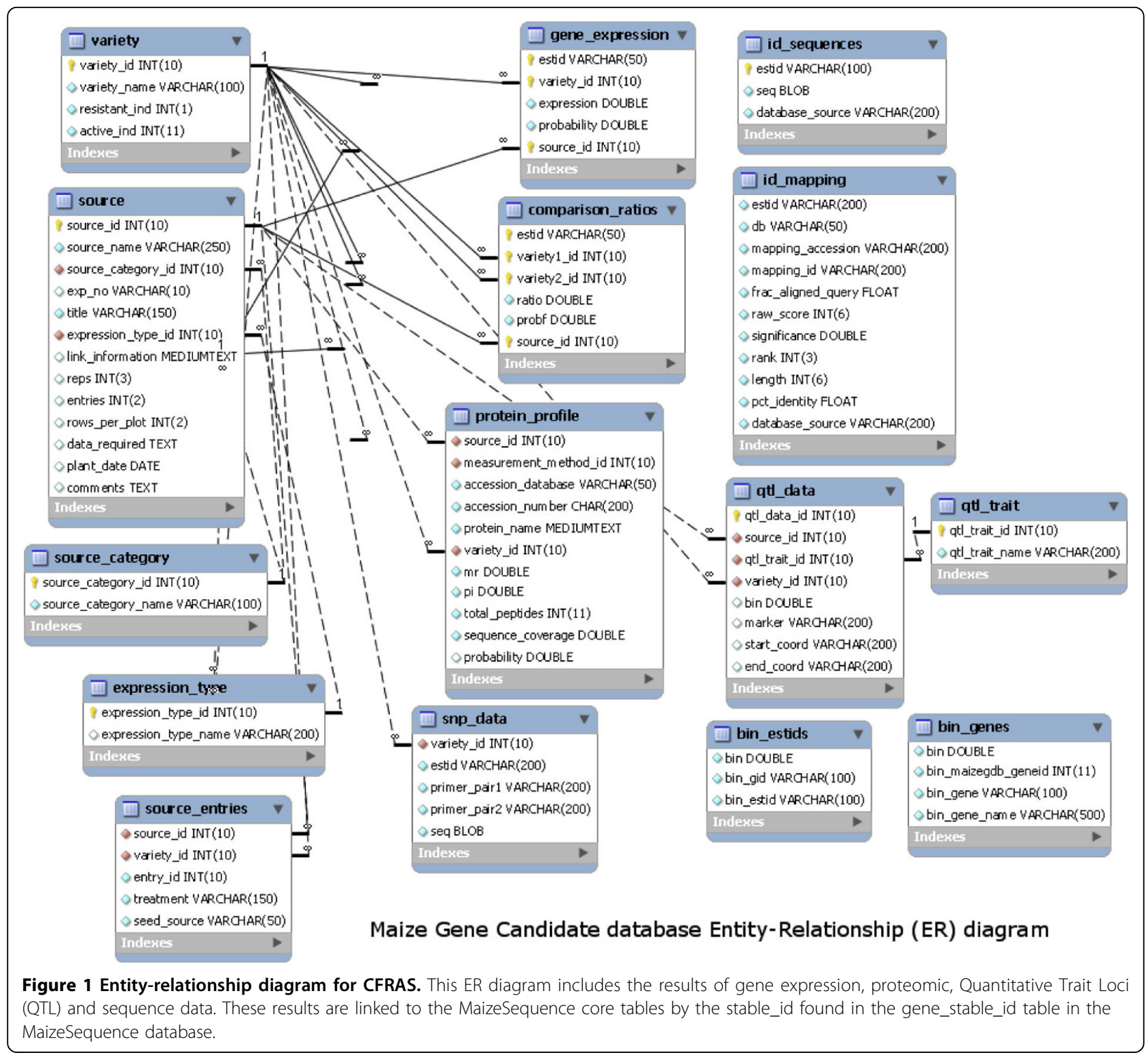

The web interface is implemented in Perl CGI. The database contains data from the maize/Aspergillus research community (Table 1 ) and from external sources (Table 2). Tables 1 and 2 illustrate the many different types of data that must be integrated. Our strategy has been to develop procedures for mapping all of the identifiers/sequences to the newly released maize genome. Procedures for remapping as the maize genome is refined have also been developed. We map both EST and proteins sequences to the MaizeSequence sequences using Exonerate. With a requirement of $80 \%$ or greater similarity, more than $99 \%$ of the 28,000 EST sequences and more than $99.5 \%$ of the 715 unique protein sequences map to unique genes in the maize genome.
The strategy for mapping Expression Sequence Tag (EST) data and protein data to the maize genome is as follows:

1) Load the MaizeSequence core tables. The version currently loaded is MySQL core_53_4a (http://ftp.maizesequence.org/current/databases/zea_mays_core_53_4a. sql.gz).

2) Map EST and protein identifiers to the maize sequence using Exonerate and requiring 80\% similarity. [18].

3) Retrieve gene model annotation from the MaizeSequence core MySQL relational tables.

4) Link gene model annotations to AgBase functional annotations [19] 


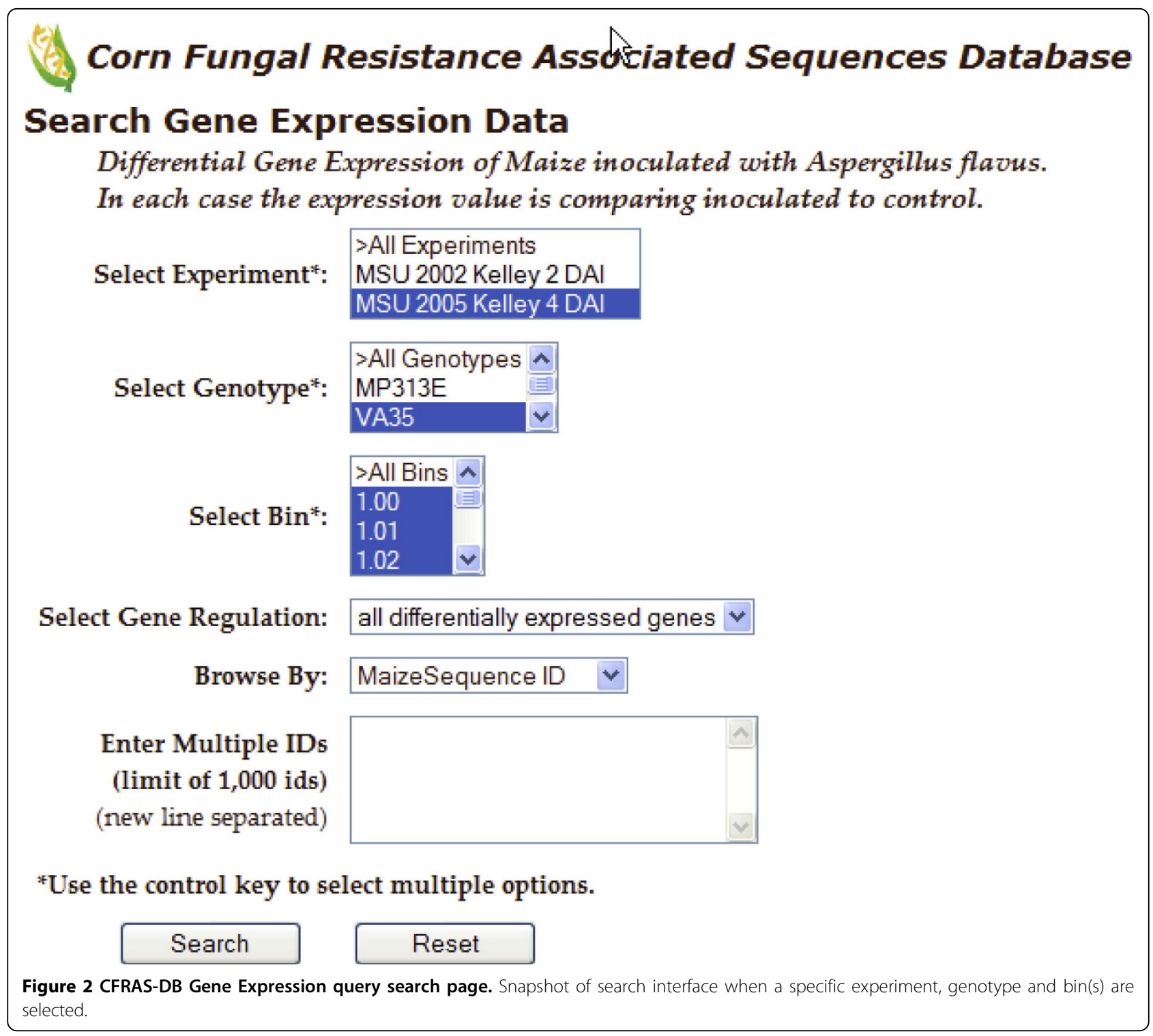

5) Link gene model annotations to bin using MaizeGDB bin coordinates [20].

In addition to sequence data and annotations, we also integrate extensive metadata describing the experiments including date, location, the genotypes used, number of days after inoculation when samples were collected, and the tissue used in the experiments. Gene expression data is included for comparison of both control and inoculated samples and for comparison of different genotypes. All microarray data is accompanied with the metadata required for submission to the GEOarchive [21].

The gene centric design of CFRAS-DB is reflected in the entity-relationship diagram for the core CFRAS-DB tables (Figure 1). The gene_stable_id table provides the link between the MaizeSequence data and the CFRAS-DB data. CFRAS-DB currently consists of twenty-five tables excluding the MaizeSequence core tables. Both EST identifiers and UniProt identifiers are mapped to MaizeSequence gene identifiers and a procedure for remapping has been established as MaizeSequence is updated. Because of the complexity of identifier mapping, we do not support automatic upload of data into the system. We do encourage members of the corn fungal resistance community to submit data for inclusion in the database. We accept gene expression data in the GEO format and proteomics data in the PRIDE format. We will work with scientists to obtain all the necessary metadata.

\section{Data sources}

\section{QTL data}

Analysis of quantitative trait loci (QTL) from resistant lines has successfully identified dozens of QTL, mainly 


\begin{tabular}{|c|c|c|c|c|c|c|c|c|}
\hline \multicolumn{9}{|c|}{$\begin{array}{l}\text { Search Gene Expression Results } \\
\text { Differential Gene Expression of Maize inoculated with Aspergillus flavus. In each case the expression value is } \\
\text { comparing inoculated to control }\end{array}$} \\
\hline Previous & Go to page & 1 & of 11 & Next & Return search & Reset & Export Results & \multirow[b]{2}{*}{ Probability } \\
\hline ESTID & \multicolumn{2}{|c|}{ MaizeSequence ID } & Bin & \multicolumn{2}{|c|}{ Experiment } & \multicolumn{2}{|r|}{ Fold Change } & \\
\hline AI438624 & GRMZM? & 5139963 & $\underline{1.02}$ & MSI & 2005 Kelley 4 DAI & VA3: & 1.390 ] & 0.007 \\
\hline AI649478 & GRMZM & 5016511 & 1.02 & MSI & 2002 Kelley 2 DAI & VA3: & 0.651 & 0.047 \\
\hline AI834747 & GRMZMZ & G420357 & 1.02 & MS & 2005 Kelley 4 DAI & VA3: & $1.351 \mathrm{I}$ & 0.005 \\
\hline AI 964459 & GRMZMZ & 9410704 & 1.02 & MSI & 2005 Kelley 4 DAI & VA3: & $0.724 I$ & 0.009 \\
\hline AW060056 & GRMZM2 & 5000686 & 1.02 & MS & 2002 Kelley 2 DAI & VA3: & 2.0021 & 0.022 \\
\hline AW231407 & GRMZMZ & 5069765 & 1.02 & MSI & 2002 Kelley 2 DAI & VA3: & 1.8331 & 0.045 \\
\hline AZM4 100347 & GRMZM & 5008122 & 1.01 & MS & 2005 Kelley 4 DAI & VA3: & $0.591-$ & 0.034 \\
\hline AZM4 101300 & GRMZMZ & 5025642 & 1.01 & MSI & 2005 Kelley 4 DAI & VA3: & $0.392+$ & 0.045 \\
\hline AZM4 101601 & GRMZM & 4436226 & $\underline{1.01}$ & MS & 2005 Kelley 4 DAI & VA3: & 0.677 & 0.046 \\
\hline AZM4 101649 & GRMZM? & 6163116 & 1.02 & MSI & 2005 Kelley 4 DAI & VA3: & 0.590 & 0.015 \\
\hline
\end{tabular}

Figure 3 Results generated from the query shown in Figure 2. From this results list, the user can select any ESTID, MaizeSequence ID, bin, experiment and genotype for further details. The user can also sort the results based on any column in the results page.

\section{CFRAS-DB MaizeSequence Detail for GRMZM2G139963}

\begin{tabular}{llll}
\hline Gene Information & & \\
ID & Type & Bin & Location \\
GRMZM2G139963 & protein_coding & 1.02 & Chromosome 1:14,898,344-14,901,300 reverse strand
\end{tabular}

\section{Transcript Information}

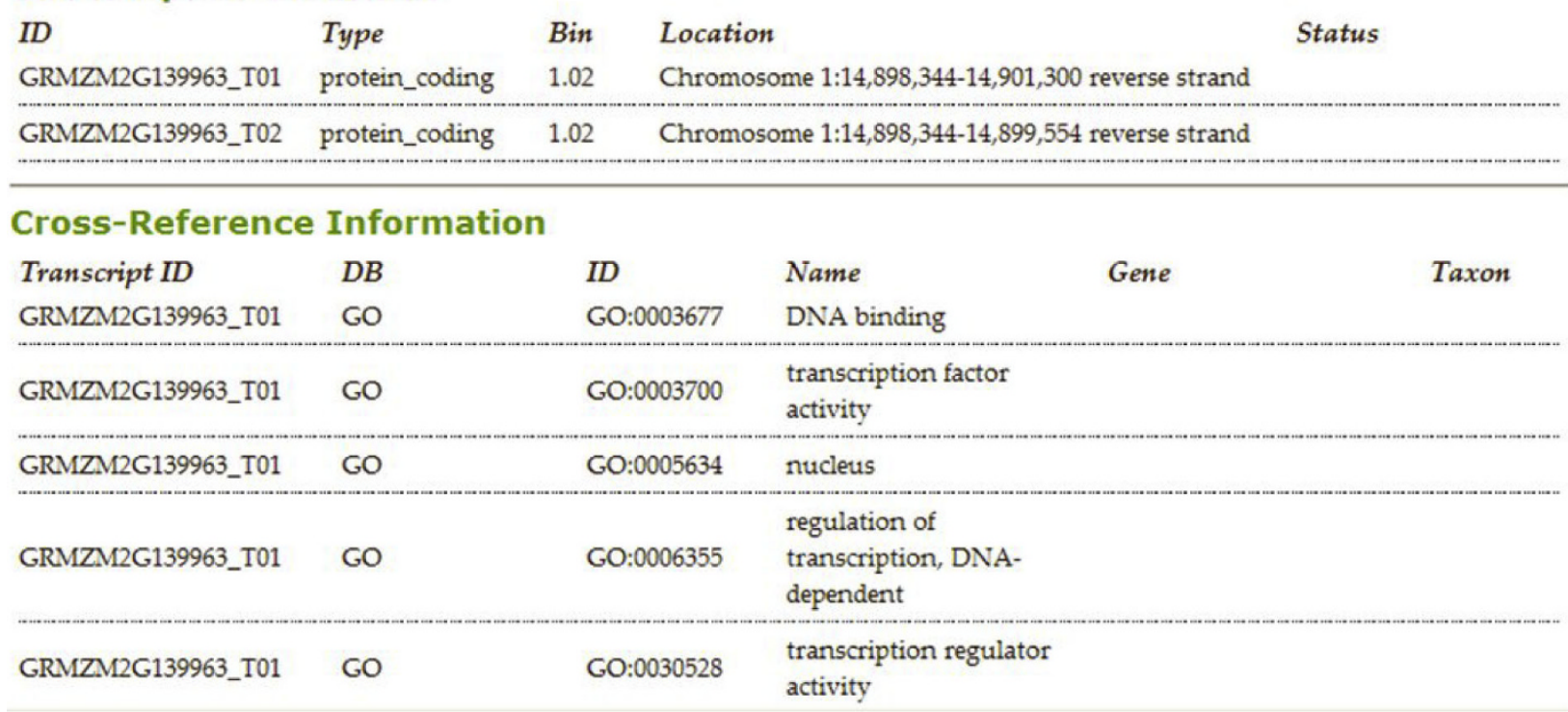

Figure 4 Details generated when a gene MaizeSequence ID is selected. Information from the MaizeSequence core tables is displayed. 
Table 3 CFRAS-DB currently supported queries

\begin{tabular}{l}
\hline Query Name \\
Gene Expression \\
Pairwise Genotypic Differential Expression \\
SNPs in Candidate Genes \\
QTL Study Data \\
Mapped QTLs by Genotype \\
Protein Profile Data \\
Combined search experiment data and gene data by genotype \\
Bin Contents \\
Combined location search for Gene Expressed data with QTL data \\
Gene Summary Counts
\end{tabular}

from the following lines: Mp715 [22], Mp717 [23], Mp313E [24], Mp420, Tx6 [25,26], Tx601, and Oh516 [27]. Each QTL tends to express only in some environments, and each line tends to contain only some of the QTL. Furthermore, very few of the QTL have a large effect, although the combined effect of several QTL can confer a high degree of resistance on a line. Some QTL that are constant over different sources or those expressed in multiple environments can be found, especially some large effect QTL on chromosome 4 (bins 4.06 and 4.08). Data from a number of QTL experiments is incorporated in CFRAS-DB.

\section{Gene expression studies of maize (Zea mays L.) ears}

The maize inbred lines Va35, Mp313E were used in the 2 days after inoculation (DAI) [28] microarray study while the inbred lines Va35, B73, Mp313E and Mp04:86 were used in the 4DAI microarray study. Va35 and B73 are inbred lines susceptible to aflatoxin and, Mp313E and Mp04:86 are inbred lines resistant to aflatoxin.

The slides used were the maize Unigene [29] 1-1.05 arrays purchased from the National Science Foundation (NSF) Maize Gene Discovery Project (MGDP). All recommendations of the minimum requirements for a microarray experiment (MIAME) checklist [30] were followed.

Tools from AgBase (http://www.agbase.msstate.edu) and SAS Version 9.1.3 (SAS, Cary, North Carolina) were used for analysis of the data and gene ontology (GO) annotation for the differentially expressed transcripts [19]. The Agbase GoSlimViewer tool was also used to produce high-level summaries of the annotations using the Plant Go Slim available from the Gene Ontology website (http://www.geneontology.org/GO.slims.shtml).

\section{Proteome profile of the developing maize (Zea mays L.) rachis}

Rachis from 21-day-old field-grown maize genotype Mp313E was profiled. For 2-DE rachis proteins were extracted with modified phenol-based protocol [31] and separated via 2-DE on 24-cm NL pH gradient 3-10 IPG strips (BioRad, Hercules, CA) and large format gels (25 $\mathrm{cm} \times 20.5 \mathrm{~cm} \times 1.5 \mathrm{~mm}$ ) slab gels with $10-15 \%$ polyacrylamide gradient. Gel pots were trypsin-digested, analyzed via 1-D LC ESI MS/MS and proteins were identified against UniProt database using ProteoIQ 1.3.01 (Bioinquire, Athens GA) software. For 2-D LC, rachis proteins were extracted using 3 procedures: modified phenol extraction [31], extraction from cell debris with chaotrops and CHAPSO and DDF (Differential Detergent Fractionation) [32]. Complex protein mixtures were in-solution digested with trypsin and analyzed via 2-D LC ESI MS/MS. Proteins were identified using ProteoIQ 1.3.01 (Bioinquire) software and UniProtKB database [33].

\section{Single Nucleotide Polymorphisms}

DNA was extracted using the CTAB method from frozen, freeze-dried leaf tissue. DNA was extracted from multiple resistant and susceptible lines. Primers were designed for each gene by using the EST sequence obtained from NCBI (http://www.ncbi.nlm.nih.gov/). Primer 3 software v. 0.40 (http://frodo.wi.mit.edu/primer3/) was used to design the primer pairs, and primers were ordered from Sigma - Genosys. Primers were verified using PCR amplification and PCR products were visualized on a $1.5 \%$ agarose gel stained with ethidium bromide.

PCR products were purified using Qiagen QIAquickTM PCR Purification Kit (Qiagen Inc.; Valencia, $\mathrm{CA})$, and sequencing reactions were prepared using Big Dye ${ }^{\bullet}$ chemistry from Applied Biosystems Inc. (Foster City, CA). The sequencing reactions were then analyzed using an Applied Biosystems 3730xl DNA Analyzer. Sequencing was performed on multiple resistant and susceptible maize genotypes, and alignment of sequences was performed using DNAMAN software v. 5.2.9 (Lynnon Corporation; Pointe-Claire, Quebec, Canada).

\section{Future directions and conclusions}

We are in the process of integrating three additional proteomics datasets and an RNASeq dataset into CFRAS-DB. The addition of RNASeq data will significantly increase the size of the database. We plan to develop a prioritization algorithm that will rank genes by the number of experiments where they were found to be differentially expressed between resistant and susceptible lines, that map to QTL known to be involved in aflatoxin accumulation resistance, have been reported in the literature to be involved in aflatoxin accumulation resistance, and that contain genes or domains known to be involved in pathways that do not favor the production of aflatoxin. 
The proteins and gene sequences identified via the queries can be investigated with pathway analyses to find larger patterns indicating major mechanisms of resistance to fungal infection or aflatoxin accumulation in maize. The reports generated by the queries can be formatted for automatic update into network analysis programs. In addition to maize genetics, genomics, proteomics, and biochemical studies, data from different $A$. flavus strains can be added, and perhaps resistance data from maize infected with other fungal strains as well. Information on environment data (from field phenotyping studies) will allow more complexity to be added to the analyses, which is important considering the complexity of the trait under study.

The CFRAS database and the associated web-based interface allow researchers to examine many lines of evidence to assess the potential role of a gene or group of genes in the response of different maize lines to A. flavus infection and subsequent production of the toxin, aflatoxin, by the fungus. CFRAS contains data from QTL experiments, gene expression studies, proteomics studies (both gel-based and shotgun), as well as sequence data from the literature. The database also contains results by other research groups who have identified genes potentially involved in resistance or susceptibility to A. flavus, and we expect more will follow. The web-based interface provides researchers with the capability to mine the database across many different experiments.

\section{Availability and requirements}

The database is publicly available from the AgBase main page (http://www.agbase.msstate.edu). Researchers wishing to submit data to CFRAS-DB should contact cfrasdb@cse.msstate.edu. Access to the restricted database can be made available to submitters prior to publication of their results to facilitate analysis.

\footnotetext{
Acknowledgements

This paper was approved for publication as Journal Article No. J-11809 for Mississippi Agricultural and Forestry Experiment Station, Mississippi State University. This research was funded through specific cooperative agreement with the USDA-ARS Corn Host Plant Resistance Research Unit (SCA 58-64066-039). Partial funding was also provided by NSF EPS-0903787. Mention of trade names or commercial products in this publication is solely for the purpose of providing specific information and does not imply recommendation or endorsement by the USDA.

This article has been published as part of BMC Bioinformatics Volume 11 Supplement 6, 2010: Proceedings of the Seventh Annual MCBIOS Conference. Bioinformatics: Systems, Biology, Informatics and Computation. The full contents of the supplement are available online at http://www.biomedcentral.com/1471-2105/11?issue=S6.
}

\section{Author details}

${ }^{1}$ Department of Biochemistry and Molecular Biology, Mississippi State University, MS, USA. ²Department of Computer Science and Engineering, Mississippi State University, MS, USA. ${ }^{3}$ Institute of Digital Biology, Mississippi State University, MS, USA. ${ }^{4}$ Corn Host Plant Resistance Research Unit, USDA/
ARS, Mississippi State, MS, USA. ${ }^{5}$ Department of Biology, Villanova University, Villanova, PA, USA. 'ife Sciences and Biotechnology Institute, Mississippi Agricultural and Forestry Experiment Station, Mississippi State University, MS, USA. ${ }^{7}$ Department of Crop and Soil Sciences, The Pennsylvania State University, PA, USA. ${ }^{8}$ Department of Human Genetics, Emory University of Medicine, GA, USA. ${ }^{9}$ Department of Plant and Soil Sciences, Mississippi State University, MS, USA.

\section{Authors' contributions}

RYK facilitated the discussion on the database, led the writing effort, helped develop the requirements of the database, and conducted the gene expression studies. JWH designed and created the initial database. MLW helped design the structure of the database, co-wrote and edited the article, and did the QTL studies and meta-analysis. CG and SMB designed the database structure, developed the id mapping approach implemented the database and the web-based interface, and co-wrote the article. LKH helped design the structure of the database, co-wrote and edited the article. OP and TP performed proteomic analysis of maize rachis and contributed to writing the article. BP performed proteomic analysis of maize silk. DSL led the proteomic studies and provided guidance. JEM aided in gene expression studies and conducted the SNP studies. AKA aided in gene expression studies, and contributed to data extraction. SO reviewed published literature and manually curated the references to select candidate genes. WBH helped specify the requirements for the database and edited the manuscript. WPW initiated, planned, and oversaw the QTL and gene expression studies and assembled the group of investors working on the database project.

\section{Competing interests}

The authors declare that they have no competing interests.

Published: 7 October 2010

\section{References}

1. Food and Agricultural Organization of the United Nations: Manual on the application of the HACCP system in mycotoxin prevention and control. FAO Food and Nutrition Rome: Food and Agriculture Organization of the United Nations 2001 [http://www.fao.org/docrep/005/y1390e/y1390e00. htm], Paper 73.

2. Larson E: Minimizing Aflatoxin in Maize. Information sheet 1563 Mississippi State, Mississippi: Mississippi State University Extension Service 2002 [http:// msucares.com/pubs/infosheets/is1563.htm].

3. Rodriguez LADel Bosque: Impact of agronomic factors on aflatoxin contamination in preharvest field maize in northeastern Mexico. Plant Dis 1996, 8:988-993.

4. Payne GA: Aflatoxin in Maize. Crit Rev Plant Sci 1992, 10:423-440.

5. Payne GA, Brown MP: Genetics and physiology of aflatoxin biosynthesis. Annu Rev Phytopathol 1998, 36:329-362.

6. Betran FJ, Isakeit T: Aflatoxin accumulation in maize hybrids of different maturities. Agronomy J 2004, 96:565-570.

7. Clements MJ, White DG: Identifying sources of resistance to aflatoxin and fumonisin contamination in corn grain. J. Toxicology 2004, 23:381-396.

8. Windham GL, Williams WP: Aspergillus flavus infection and aflatoxin accumulation in resistant and susceptible maize hybrids. Plant Dis 1998 82:281-284

9. Cleveland TE, Dowd PF, Desjardins AE, Bhatnagar D, Cotty PJ: United States Department of Agriculture-Agricultural Research Service research on pre-harvest prevention of mycotoxins and mycotoxigenic fungi in US crops. Pest Manag Sci 2003, 59:629-642.

10. U.S. Food and Drug Administration: Mycotoxins in domestic and imported foods CPG 7307.001. [http://www.fda.gov/downloads/Food/ GuidanceComplianceRegulatoryInformation/ComplianceEnforcement/ ucm073294.pdf]

11. Van Egmond HP, Jonker MA: Worldwide regulations for mycotoxins in food and feed in 2003. Rome: Food and Agriculture Organization of the United Nations 2004 [http://www.fao.org/docrep/007/y5499e/y5499e00. htm].

12. Williams WP, Windham GL, Buckley PM: Enhancing maize germplasm with resistance to aflatoxin contamination. J Toxicology-Toxin Rev 2003, 22:175-193. 
13. Betran FJ, Isakeit T, Odvody G: Aflatoxin accumulation of white and yellow maize inbreds in diallel crosses. Crop Sci 2002, 42:1894-1901.

14. Scott GE, Zummo N: Registration of Mp313E parental line of maize. Crop Sci 1990, 30:1378.

15. Scott GE, Zummo N: Registration of Mp420 germplasm line of maize. Crop Sci 1992, 32:1296

16. Williams WP, Windham GL: Registration of maize germplasm line Mp715. Crop Sci 2001, 41:1374-1375.

17. Schnable PS, Ware D, et al: The B73 Maize Genome: Complexity, Diversity, and Dynamics. Science 2009, 326:1112-1115.

18. Slater $G$, Birney E: Automated generation of heuristics for biological sequence comparison. BMC Bioinformatics 2005, 6:31.

19. McCarthy FM, Wang N, Magee GB, Nanduri B, Lawrence ML, Camon EB, Barell DG, Hill DP, Dolan ME, Williams WP, Luthe DS, Bridges SM, Burgess SC: AgBase: a functional genomics resource for agriculture. BMC Genomics 2006, 7:229.

20. Lawrence CJ, Harper LC, Schaeffer ML, Sen TZ, Seigfried TE, Campbell DA: MaizeGDB: The Maize Model Organism Database for Basic, Translational, and Applied Research. Int J Plant Genomics 2008, 496957.

21. Barrett T, Troup DB, Wilhite SE, Ledoux P, Rudnev D, Evangelista C, Kim IF, Soboleva A, Tomashevsky M, Marshall KA, Phillippy KH, Sherman PM, Muertter RN, Edgar R: NCBI GEO: archive for high-throughput functional genomic data. Nucleic Acids Res 2009, 37:D885-890.

22. Warburton ML, Brooks TD, Windham GL, Williams WP: Identification of novel QTL Contributing Resistance to Aflatoxin Accumulation in Maize. Mol Breed

23. Warburton ML, Brooks TD, Krakowsky MD, Shan X, Windham GL, Williams WP: Identification and mapping of new sources of resistance to aflatoxin accumulation in maize. Crop Sci 2009, 49:1403-1408.

24. Brooks TD, Williams WP, Windham GL, Willcox MC, Abbas HK: Quantitative trait loci contributing resistance to aflatoxin accumulation in maize inbred Mp313E. Crop Sci 2005, 45:171-174.

25. Hamblin AM, White DG: Inheritance of resistance to Aspergillus ear rot and aflatoxin production of corn from Tx6. Phytopathology 2000, 90:292-296.

26. Paul C, Naidoo G, Forbes A, Mikkilineni V, White D, Rocheford T: Quantitative trait loci for low aflatoxin production in two related maize populations. Theor Appl Genet 2003, 107:263-270.

27. Busboom KN, White DG: Inheritance of resistance to aflatoxin production and Aspergillus ear rot of corn from the cross of inbreds B73 and Oh516. Phytopathology 2004, 94:1107-1115.

28. Kelley RY, Williams WP, Mylroie JE, Boykin DL, Hawkins LK, Windham GL, Brooks TD, Bridges SM, Scheffler BE, Wilkinson JE: Genomic Profile of maize response to Aspergillus flavus infection. Toxin Rev 2009, 28:129-141.

29. Pontius JU, Wagner L, Schuler GD: UniGene: a unified view of the transcriptome. The NCBI Handbook. Bethesda (MD): National Center for Biotechnology Information 2003, Chapter 21.

30. Brazma A, Hingam P, Quackenbush J, Sherlock G, Spellman P, Stoeckert C, Aach J, Ansorge W, Ball CA, Causton HC, Gaasterland T, Glenisson P, Holstege FCP, Kim IF, Markowitz V, Matese JC, Parkinson H, Robinson A, Sarkans U, Schulze-Kremer S, Stewart J, Taylor R, Vilo J, Vingron M: Minimum information about a microarray experiment (MIAME)-toward standards for microarray data. Nat Genet 2001, 29:365-375.

31. Hurkman WJ, Tanaka C: Solubilization of plant membrane proteins for analysis by two-dimensional gel electrophoresis. Plant Physiol 1986, 81:802-806.

32. McCarthy FM, Burgess SC, Van den Berg BHJ, Koter MD, Pharr GT: Differential Detergent Fractionation for non-electrophoretic eukaryote cell proteomics. J Proteome Res 2005, 4:316-324.

33. Pechanova O, Pechan T, Ozkan S, McCarthy FM, Williams PW, Luthe DS: Proteome profile of the developing maize (Zea mays L.) rachis. Proteomics 2010

doi:10.1186/1471-2105-11-S6-S25

Cite this article as: Kelley et al.: Integrated database for identifying candidate genes for Aspergillus flavus resistance in maize. BMC Bioinformatics 2010 11(Suppl 6):S25.

\section{Submit your next manuscript to BioMed Central and take full advantage of:}

- Convenient online submission

- Thorough peer review

- No space constraints or color figure charges

- Immediate publication on acceptance

- Inclusion in PubMed, CAS, Scopus and Google Scholar

- Research which is freely available for redistribution

Submit your manuscript at www.biomedcentral.com/submit 